\title{
NUMERICAL INVESTIGATION OF THE DYNAMIC RESPONSES OF COMPOSITE MATERIAL SUBJECTED TO BUBBLE COLLAPSE
}

\author{
XIAOJIAN MA, BIAO HUANG \& GUOYU WANG \\ School of Mechanical and Vehicular Engineering, Beijing Institute of Technology, China
}

\begin{abstract}
The presence of composite materials is an alternative solution to effectively overcome the troubles caused by the occurrence of bubble collapse in the marine propulsion systems, such as noise, vibration, pressure pulsations, and heavy erosion. The objective of this paper is to apply computational method to investigate the deformation of composite material with clamped ends subjected to the pressure loads caused by a 2D single bubble with a non-spherical collapse. The Euler-Lagrangian numerical method is supposed to simulate the bubble shape evolution, the transient pressure loads, and the dynamic response between fiber layers. With emphasis on numerical method, the CLSVOF method is used to track the air-liquid interface and the Ghost Fluid method is adopted to simulate the incompressible flow. The numerical results reveal that the substantial increase in pressure fluctuations on the composite material boundary are caused by the bubble evolution process near the wall, involving the motion of bubble rising to the boundary, the formation of the re-entrant jet, and the splitting of the daughter bubble. The impact pressure load significantly contributes to the deformation of the composite material. Furthermore, the effect of standoff distance between the bubble and wall on the dynamic responses among the fiber layers of composite material boundary is preliminarily discussed.
\end{abstract}

Keywords: bubble collapse, high speed jet, pressure loads, composite material, carbon fiber layer, CLSVOF.

\section{INTRODUCTION}

The occurrence of cavitation in the marine propulsion systems, which are typically designed with the traditional materials such as metal and metallic alloy, can lead to many problems, such as noise [1], vibration [2], pressure pulsations [3], and heavy erosion [4]. Rayleigh [5] applied the theory of spherical bubble to interpret the cavitation phenomenon. Recently, the dynamics of bubble near the structure, especially the non-spherically collapse and the formation of liquid jet, is considered as the destructive action, involving impulsive pressure and shock wave. With the development of materials, the presence of various composite is an alternative solution to effectively overcome those troubles from bubble collapse, due to its favorable self-adaptive ability, corrosion resistance, light weight and anisotropy properties [6].

Extensive studies on dynamic behaviors of bubble collapse near the different boundary have been reported in the works of Blake et al. [7], Zhang et al. [8], Brujan et al. [9], Hung and Hwangfu [10], Lauterborn and Bolle [11] and Klaseboer et al. [12]. They found that the bubble presents different behaviors near various boundaries, for example, bubbles migrate towards the rigid boundary and away from the free surface. Based on this interesting phenomenon, many researchers paid their attention on neutral collapsing bubbles, which do not move towards or away the boundary, by changing the flexible material coated on the surface of fluid machine [13]. Shima et al. [14] used high-speed photograph to measure the effect of the surface stiffness and surface inertia of the composite materials on the growth and collapse of single bubble. They demonstrated that surface stiffness is a particularly important factor to the bubble dynamics compared with the surface inertia. And both parameters contribute to the formation of the neutral bubble collapse. Tomita and Shima [15] utilized the laser-induced 
bubble to investigate the interaction between bubbles and composite material. Their works further broaden the knowledge of bubble dynamics near the composite boundary. However, the past works mostly focus on the effect of deformed structure on the bubble, but not the structure responses subject to the bubble oscillation [16]. In the present work, both aspects will be further considered comprehensively.

Due to the complex, multi-physic, fluid-structure interaction problems, the physical interaction mechanisms between the bubble and composite are still not well understood. The direction of liquid jet and migration of bubble collapse can be predicted by the theory of Kelvin impulse, which is first proposed by Gibson and Blake [17]. The additional works about Kelvin impulse were reported by Blake et al. [18] and Best and Blake [19]. However, the theory is suitable for the condition that bubble locates near a rigid wall and free surface, but not a good predictor for elastic boundary. In the recent years, significant progress of the simulation has been made in understanding and modeling bubble collapse near the elastic boundary by advanced visualization technique and detail flow field measurements. Kalumuck et al. [20] employed the coupled finite element method (FEM) and boundary element method (BEM) to simulate the cavitation erosion caused by the bubble collapse. They found the shock wave released by the bubble can produce high stress waves, which propagate inside the wall, cause the deformation and the failure of the materials. Hsiao et al. [21] investigated the material pitting from cavitation bubble collapse by a hybrid numerical approach, which links an incompressible BEM solver and a compressible finite difference flow solver. They found the cavitation pit is formed on the material surface, when the maximum impulsive pressure exceeds the yield stress of the material. Young et al. [22] used 2D Euler-Lagrangian numerical method to investigate the fluid-structure and bubble-shock interaction during the bubble collapse near the sandwich composite structure. They quantitatively and qualitatively evaluated the influence of the Taylor's FSI effect, the bending/stretching effect, the boundary effect and the core compression effect during the bubble collapse. Detail reviews on these numerical methods have been reported in the previous work of reference [23].

Although the bubble collapse has received much attention in the past years, the effect of the bubble collapse on the composite material response is still not well understood, and hence additional studies are still needed. The objectives of the present study are:

1. Improve the understanding of the dynamic behaviors of single bubble near the composite boundary.

2. Investigate the mechanism of composite material deformation due to the bubble collapse.

\section{MATHEMATICAL FORMATION AND NUMERICAL METHOD}

To model dynamic responses of the composite material subjected to the bubble collapse, a two-step numerical scheme is adopted. Eulerian incompressible fluid equations are used for fluid regions, while the equation of motion and the material constitutive equation are needed for solid regions, which is generally solved by Lagrangian finite element method. In order to obtain the bubble shape and structure responses, the interfaces between fluid-fluid and fluidsolid should be specially treated.

\subsection{Eulerian fluid equations}

In the present study, the dynamic behavior of single bubble is investigated with the assumption of an isothermal system. Hence, the mass conservation equation for 
incompressible Newtonian fluids (for liquid and gas) can be expressed by a single fluid continuum as follows:

$$
\nabla \cdot \boldsymbol{u}=0
$$

The Navier-Stokes equation can be written as:

$$
\rho(\phi)\left(\frac{\partial \boldsymbol{u}}{\partial t}+\boldsymbol{u} \cdot \nabla \boldsymbol{u}\right)=-\nabla p+\rho(\phi) \boldsymbol{g}+\nabla \cdot\left[\mu\left(\nabla \boldsymbol{u}+\nabla^{T} \boldsymbol{u}\right)\right]+\sigma \kappa \nabla H_{\varepsilon}(\phi),
$$

in which $u$ is the velocity vector; $p$ is the pressure; $\boldsymbol{g}$ is the gravitational acceleration; $\mu$ is the viscosity of the fluid; $\sigma$ is the surface tension coefficient; $\kappa$ is the surface curvature, and $\Phi$ is the level-set (LS) function. The density $\rho$ is the mixture of liquid and gas, and the definition is $\rho=\alpha \rho_{g}+(1-\alpha) \rho_{l}$ where $\alpha$ is 1 for gas and 0 for liquid. In present works, the ghost fluid method based on numerical technique is used to capture the gas-liquid interface. The CLSVOF method is employed to capture the moving interface. The detailed information about treatment for gas-liquid interface is reported by the works of Wang et al. [24].

\subsection{Lagrangian solid equations}

For a solid structure which ignore the structural damping effects, the governing equation of motion can be written in matrix form as follows [25]:

$$
\begin{aligned}
& \boldsymbol{M} \ddot{\boldsymbol{\delta}}+\boldsymbol{K} \boldsymbol{\delta}=\boldsymbol{F}, \quad t \in] 0, t[ \\
& \boldsymbol{\delta}(0)=\boldsymbol{\delta}_{0} \\
& \dot{\boldsymbol{\delta}}(0)=\dot{\boldsymbol{\delta}}_{0}
\end{aligned}
$$

where $\delta$ and $\delta$ are the Lagrangian solid displacement and acceleration vectors, respectively. Subscript 0 represents the initial conditions. $M, K$ and $F$ are solid mass matrices, stiffness matrices and external force vector, respectively. And their function is defined as follows:

$$
\begin{gathered}
\boldsymbol{M}=\int_{\Omega} \boldsymbol{N}^{T} \rho_{S} \boldsymbol{N} d V, \\
\boldsymbol{K}=\int_{\Omega} B^{T} C B d V, \\
\boldsymbol{F}=\int_{\Omega} \boldsymbol{N} \boldsymbol{f} \mathrm{d} \boldsymbol{\Omega}+\int_{\Gamma_{\mathrm{h}}} \boldsymbol{N h} \mathrm{d} \boldsymbol{\Gamma},
\end{gathered}
$$

where $N$ is the displacement interpolation matrix and $B$ is the strain-displacement matrix. $\Omega$, $f$ and $h$ are computational domain, body force vector, and traction boundary conditions, respectively. As for the treatment about the fluid-solid interface, the Eulerian and Lagrangian solvers are coupled by an additional step of solving the fluid and solid equation of motion at the fluid-solid interface, which updates the interface status, including pressure, velocity and density, etc. 


\subsection{Fluid-solid interface treatment}

The motion of fluid-solid interface is solved by the Lagrangian finite element (FEM) solver. The fluid characteristic equation and the solid motion equation are solved and analyzed by the Eulerian fluid and Lagranian solvers, respectively. In the calculation process, the characteristic parameters of fluid domain, such as pressure fluctuation and velocity condition, are imposed on the fluid-solid interface. Compared with the Lagrangian FEM elements used in solid domain, two different Eulerian cells are employed in the fluid domain, namely, real and ghost fluid cells. In present work, the Eulerian fluid domain is solved by in-house feature-recognition software package by Fortran 14.0, while the Lagrangian solid domain is solved by the software of Ansys workbench 17.0 (APDL) [26].

\section{RESULTS AND DISCUSSION}

\subsection{Numerical setup}

To numerically investigate the composite material responses subjected to bubble collapse, Fig. 1 shows the schematic description of solution domain for numerical simulation. As shown in Fig. 1(a), numerical simulations of bubble dynamics near the composite material wall are performed on an axisymmetric computational domain, which includes both fluid and composite computational ones. The fluid domain with no-reflective and no-slip boundary condition is in contact with the bottom of the composite material wall with clamped ends. The fluid domain is assumed to be full of single bubble and liquid, which both are initially quiescent. The position of spherical bubble with radius $R$ locates initially below the point $O$, which is the center of the composite material wall. The vertical standoff distance between point $O$ and center of bubble is defined as parameter $d$. In addition, the parameter $L$ and $H$ are length and height of fluid domain respectively, while the parameter $b$ is the thickness of the composite wall.

In present works, the bubble radius $R$ is initially set as $1 \mathrm{~mm}$. The height of the simulation domain is set as $H=8 R$ and $L=8 R$, so that the initial bubble is sufficiently far away from the bottom wall and vertical wall [27]. To further clearly present the detail characteristics of the composite material, Fig. 1(b) shows the schematic drawing about the number and direction of the carbon fiber layers. As shown in Table 1, the density of every carbon fiber layer is $1490 \mathrm{~kg} / \mathrm{m}^{3}$, and its Young's Modulus is $0.08 \mathrm{GPa}$ in X direction and $0.12 \mathrm{GPa}$ in $\mathrm{Y}$ direction. And the composite wall is composed with four carbon fiber layers with different directions, i.e. $0,90,0$ and 90 degrees alternatively. The thickness of the composite wall $b$ is about $2 \mathrm{~mm}$ in present works.

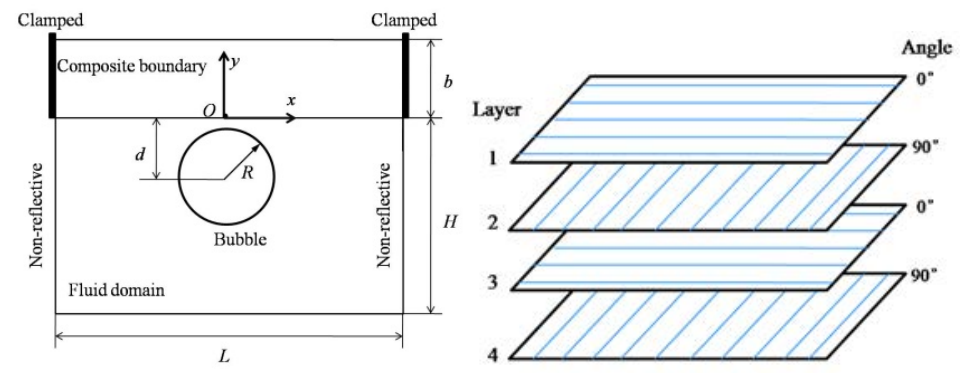

Figure 1: The schematic description for the simulated model con Figuration: $(\mathrm{a}-\mathrm{left})$ the whole calculating domains involving fluid and composite domains, $(\mathrm{b}-$ right $)$ the layers and direction of the composite material wall. 
Table 1: The detail parameters about composite structure.

\begin{tabular}{|c|c|c|c|c|c|}
\hline Items & Material & $\begin{array}{c}\text { Young's } \\
\text { Modulus } \\
(\mathrm{X})(\mathrm{GPa})\end{array}$ & $\begin{array}{c}\text { Young's } \\
\text { Modulus } \\
(\mathrm{Y})(\mathrm{GPa})\end{array}$ & $\begin{array}{c}\text { Density } \\
\left(\mathrm{kg} / \mathrm{m}^{3}\right)\end{array}$ & $\begin{array}{c}\text { Fiber layer } \\
\text { direction } \\
\left({ }^{\circ}\right)\end{array}$ \\
\hline Composite & $\begin{array}{c}\text { Carbon } \\
\text { fiber }\end{array}$ & 0.08 & 0.12 & 1490 & $0,90,0,90$ \\
\hline
\end{tabular}

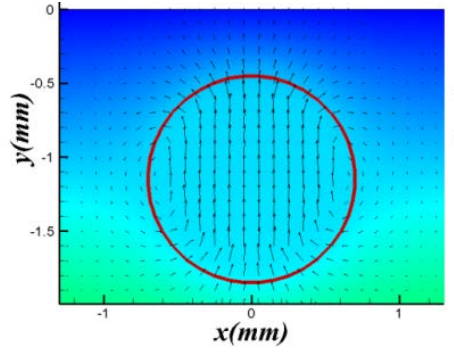

a) $\mathrm{t}=0.1 \mathrm{~ms}$

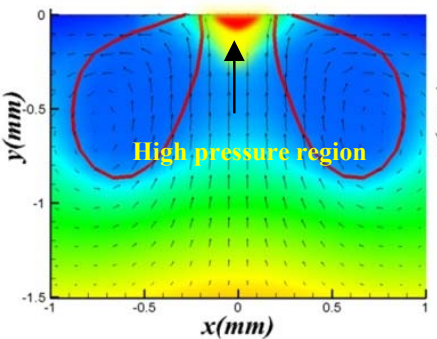

c) $t=0.5 \mathrm{~ms}$

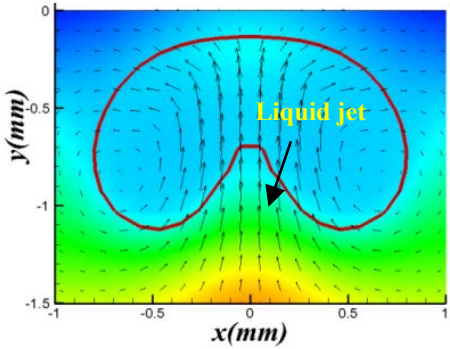

b) $\mathrm{t}=0.3 \mathrm{~ms}$

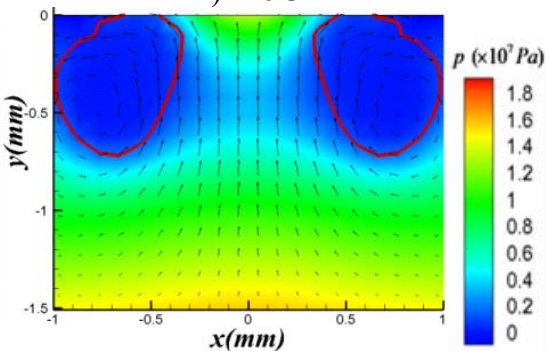

d) $t=0.7 \mathrm{~ms}$

Figure 2: The temporal evolution of bubble shapes, velocity vectors and pressure contours under the composite structure with four carbon fibers.

3.2 The interaction between bubble collapse and corresponding dynamic response of composite structure

To demonstrate the bubble dynamics near the composite wall, Fig. 2 shows the temporal evolution of bubble shapes, velocity vectors and pressure contours under the composite structure with four carbon layers, as discussed in Table 1. The numerical calculation begins from the maximum volume of the bubble to bubble collapse. As observed at $t=0.1 \mathrm{~ms}$, the spherical bubble is merged in fluid field, and the non-dimensional standoff distance between bubble and wall is about $3.0(d / R=3.0)$. Due to the effect of pressure difference between upper and lower regions caused by restrictions of the wall, the bubble has a tendency to move upward the composite wall. When $t=0.3 \mathrm{~ms}$, compared with top margin of the bubble, the bottom margin of the bubble has a faster deformation rate, due to the occurrence of the relative higher pressure at the bottom side of a bubble. Then a high-speed liquid jet is formed and impact towards to top wall. At the same time, the center of bubble vertically rises from $y=-1.5 \mathrm{~mm}$ to $-0.8 \mathrm{~mm}$. As the time increases to $t=0.5 \mathrm{~ms}$, due to the restriction of the wall, the high-speed liquid jet penetrates through the opposite side of the bubble, impacts directly on the composite wall, forms the toroidal shape and results in a highly localized pressure 
region at wall center. When the time reaches to $t=0.7 \mathrm{~ms}$, the liquid jet continues to impact on the wall due to the inertia force. But due to the increase of the width of the liquid jet, the pressure at wall center is decreasing caused by the water-hammer effect [28].

To further investigate the relationship between pressure loads and the bubble nonspherical collapse near the wall, Fig. 3 shows the temporal evolution of the impact pressure loads at the central point $O$ of the top wall boundary and the distance between two poles of the bubble. In this figure, the solid line represents the value of impact pressure load, while the dotted line shows the value of distance between two poles of bubble. The value of dotted line decreases, which means the bubble collapse rapidly and the liquid jet is generated. As observed, the peak of the impact pressure is formed by the bubble toroidal bubble collapse. The peak is founded at $t=0.4 \mathrm{~ms}$, when the distance between two poles of bubble is about zero, which indicates the high-speed liquid jet penetrates through the upper side of the bubble and impacts on the wall. In addition to this peak, the pressure fluctuation after $t=1.0 \mathrm{~ms}$ are observed due to bouncing back and forth of pressure or shock waves caused by combined effect between daughter bubbles, bubble surface and boundary wall.

To further investigate the effect of bubble collapse on the characteristics of composite material responses, the temporal evolution of composite material deformation contours is shown in Fig. 4. Notice that the composite material and its deformation have been magnified by forty times. In order to visualize the deformation of the composite wall better, the solid and black line indicate the original shape and position of the composite wall. As shown in Fig. 4, the initial deformation begins at the center of the composite wall near the wet surface when bubble rises due to pressure difference at $t=0.1 \mathrm{~ms}$. When $t=0.2 \mathrm{~ms}$, the high deformation region has a tendency to propagate from the wet surface to dry one of the composite wall and move radically away from the impact location $O$ to two clamped ends,

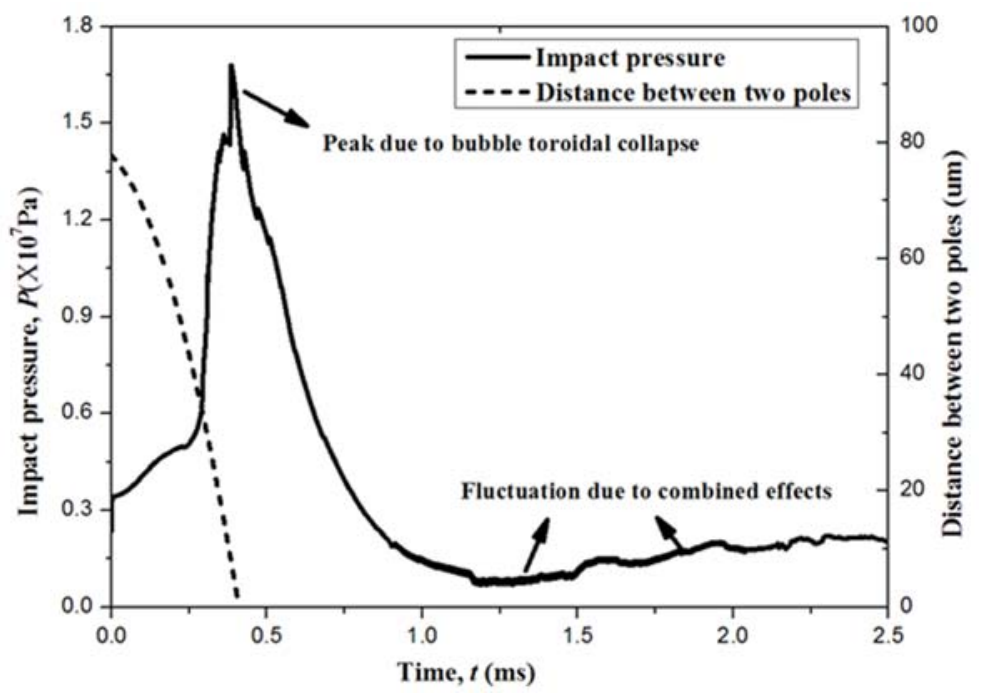

Figure 3: The temporal evolution of impact pressure loads at the central point $C$ of the top boundary and the distance between two poles of the bubble. 


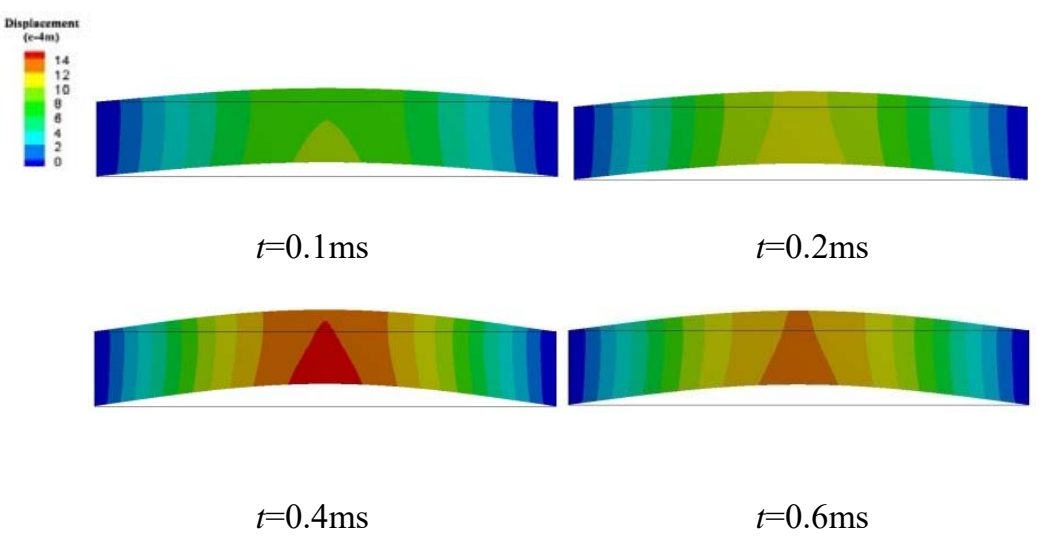

Figure 4: The temporal evolution of composite material deformation contours subjected to bubble collapse when $d / R=3.0$. Notice that the composite material and its deformation have been magnified by forty times.

respectively. As the time increases to $t=0.4 \mathrm{~ms}$ when the pressure almost reaches to the peak, the composite wall exhibits the largest upward deflection due to the impact pressure loads caused by bubble toroidal collapse. When $t=0.6 \mathrm{~ms}$, the range of high deformation decreases due to dissipation of the pressure loads and transmission of the energy through the solid deformations. On the other hand, the elastic storage force also pushes the composite wall downwards the original position from the largest displacement.

\subsection{The effect of standoff distance on the dynamic response of composite structure}

In the section 3.2, the impulsive pressure loads caused by bubble toroidal bubble collapse is founded to be closely consistent with the deformation of the composite wall. To further demonstrate the effect of the standoff distance on the composite material, Fig. 5 shows the comparisons of the composite material displacement at point $O$ under three different standoff distances between the wall and the center of bubble, namely $d / R=2.5,3.0$ and 3.5. The black line represents the standoff distance $d=2.5$ and the red line is case under $d / R=3.0$, while the blue line shows the $d / R=3.5$. As shown in Fig. 5, with the value of $d / R$ increases, the displacement at point $O$ decreases significantly. When the bubble centroid is far away from the composite wall, the impulsive pressure loads maybe dissipate during the energy propagation. As a result, the impulsive pressure loads are highly dependent on the initial standoff distance between the bubble and composite boundary. On the other hand, the structure responses also are consistent with bubble size and fluid characteristics, as well as the composite materials, such as the fiber angles and layers [29].

\section{CONCLUSIONS}

The Euler-Lagrangian numerical method is applied to simulate deformation of composite material with clamped ends subjected to the pressure loads caused by single bubble nonspherical collapse. With emphasis on numerical method, the CLSVOF method, which 


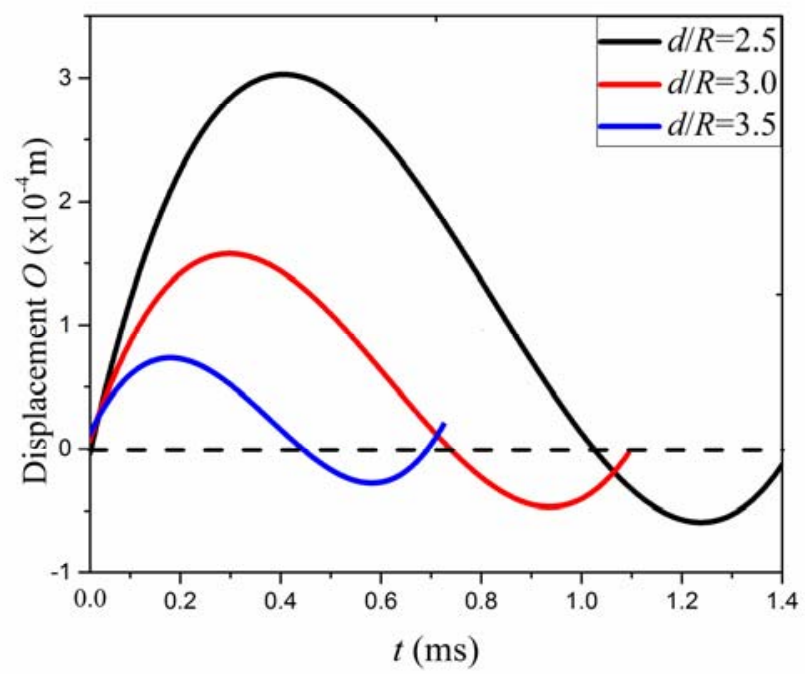

Figure 5: The comparisons of the composite material displacement at point $O$ under different standoff distance between the wall and the center of bubble.

satisfies the mass conservation and continuous surface force/stress, is used to track the air-liquid interface. The primary findings include:

a) The bubble near the composite boundary behaves in non-spherical collapse. The motion of bubble rising to the boundary, the formation of the re-entrant jet, and the splitting of the daughter bubble, may lead to substantial increase in pressure loads fluctuations on the composite material boundary.

b) The impact pressure loads significantly contribute to the deformation of the composite material. The relatively high deformation appears at the center of the composite wall near the wet surface, and then propagates from the wet surface to dry one of the composite walls and move radically away from the impact location to the clamped end. After the peak of the impact pressure loads caused by bubble toroidal collapse, the range of high deformation decreases due to dissipation of the pressure loads and transmission of the energy through solid deformations.

c) The impulsive pressure loads are highly dependent on the initial standoff distance between the bubble and composite boundary. With the distance between bubble and wall increases, the reflection of the composite wall decreases significantly. It is inferred that the impulsive pressure loads maybe dissipate during the energy propagation, when the bubble centroid is far away from the composite wall.

\section{ACKNOWLEDGEMENTS}

The authors gratefully acknowledge the support by the National Natural Science Foundation of China (Grant Nos.: 51679005 and 51479002), National Natural Science Foundation of Beijing (Grant No.: 3172029), and the Open Foundation of State Key Laboratory of Hydraulic and Mountain River Engineering (Sichuan University, China). 


\section{REFERENCES}

[1] Ma, X., Huang, B., Wang, G. \& Zhang, M., Experimental investigation of conical bubble structure and acoustic flow structure in ultrasonic field. Ultrasonics Sonochemistry, 34, pp. 164-172, 2017

[2] Huang, B., Young, Y.L., Wang, G. \& Shyy, W., Combined experimental and computational investigation of unsteady structure of sheet/cloud cavitation. Journal of Fluids Engineering, 135(7), pp. 71-301, 2013.

[3] Senocak, I. \& Shyy, W., A pressure-based method for turbulent cavitating flow computations. Journal of Computational Physics, 176(2), 363-383, 2002.

[4] Dular, M., Bachert, R., Stoffel, B. \& Širok, B., Experimental evaluation of numerical simulation of cavitating flow around hydrofoil. European Journal of MechanicsB/Fluids, 24(4), pp. 522-538, 2005.

[5] Rayleigh, L., On the pressure developed in a liquid during the collapse of a spherical cavity. The London, Edinburgh, and Dublin Philosophical Magazine and Journal of Science, 34(200), pp. 94-98, 1917.

[6] Wu, Q., Huang, B., Wang, G. \& Gao, Y., Experimental and numerical investigation of hydroelastic response of a flexible hydrofoil in cavitating flow. International Journal of Multiphase Flow, 74, pp. 19-33, 2015.

[7] Blake, J.R., Robinson, P.B., Shima, A. \& Tomita, Y., Interaction of two cavitation bubbles with a rigid boundary. Journal of Fluid Mechanics, 255, pp. 707-721, 1993.

[8] Zhang, A.M., Cui, P., Cui, J. \& Wang, Q.X., Experimental study on bubble dynamics subject to buoyancy. Journal of Fluid Mechanics, 776, pp. 137-160, 2015.

[9] Brujan, E.A., Keen, G.S., Vogel, A. \& Blake, J.R., The final stage of the collapse of a cavitation bubble close to a rigid boundary. Physics of Fluids, 14(1), pp. 85-92, 2002.

[10] Hung, C.F. \& Hwangfu, J.J., Experimental study of the behaviour of mini-charge underwater explosion bubbles near different boundaries. Journal of Fluid Mechanics, 651, pp. 55-80, 2010.

[11] Lauterborn, W. \& Bolle, H., Experimental investigations of cavitation bubble collapse in the neighborhood of a solid boundary. Journal of Fluid Mechanics, 72(02), pp. 391399, 1975.

[12] Klaseboer, E., Hung, K.C., Wang, C., Wang, C.W., Khoo, B.C. \& Boyce, P., Experimental and numerical investigation of the dynamics of an underwater explosion bubble near a resilient/rigid structure. Journal of Fluid Mechanics, 537, pp.387-413, 2005.

[13] Brujan, E.A., Nahen, K., Schmidt, P. \& Vogel, A., Dynamics of laser-induced cavitation bubbles near elastic boundaries: influence of the elastic modulus. Journal of Fluid Mechanics, 433, pp. 283-314, 2001.

[14] Shima, A., Tomita, Y., Gibson, D.C. \& Blake, J.R., The growth and collapse of cavitation bubbles near composite surfaces. Journal of Fluid Mechanics, 203, pp. 199$214,1989$.

[15] Tomita, Y. \& Shima, A., Mechanisms of impulsive pressure generation and damage pit formation by bubble collapse. Journal of Fluid Mechanics, 169, pp. 535-564, 1986.

[16] Gong, S.W. \& Klaseboer, E., Interaction between collapsing bubble and viscoelastic solid: Numerical modelling and simulation. Applied Mathematical Modelling, 40(7), pp. 4746-4764, 2016.

[17] Gibson, D.C. \& Blake, J.R., The growth and collapse of bubbles near deformable surfaces. Applied Scientific Research, 38(1), pp. 215-224, 1982.

[18] Blake, J.R., Taib, B.B. \& Doherty, G., Transient cavities near boundaries. Part 1. Rigid boundary. Journal of Fluid Mechanics, 170, pp. 479-497, 1986. 
[19] Best, J.P. \& Blake, J.R., An estimate of the Kelvin impulse of a transient cavity. Journal of Fluid Mechanics, 261, pp. 75-93, 1994.

[20] Kalumuck, K.M., Duraiswami, R. \& Chahine, G.L., Bubble dynamics fluid-structure interaction simulation by coupling fluid BEM and structural FEM codes. Journal of Fluids and Structures, 9(8), pp. 861-883, 1995.

[21] Hsiao, C.T., Jayaprakash, A., Kapahi, A., Choi, J.K. \& Chahine, G.L., Modelling of material pitting from cavitation bubble collapse. Journal of Fluid Mechanics, 755, pp. 142-175, 2014.

[22] Young, Y.L., Liu, Z. \& Xie, W., Fluid-structure and shock-bubble interaction effects during underwater explosions near composite structures. Journal of Applied Mechanics, 76(5), pp. 51-303, 2009.

[23] Liao, Y. \& Lucas, D., A literature review of theoretical models for drop and bubble breakup in turbulent dispersions. Chemical Engineering Science, 64(15), pp. 33893406, 2009.

[24] Wang, Z., Li, Y., Huang, B. \& Gao, D., Numerical investigation on the influence of surface tension and viscous force on the bubble dynamics with a CLSVOF method. Journal of Mechanical Science and Technology, 30(6), pp. 2547-2556, 2016.

[25] Xie, W., Liu, Z., \& Young, Y.L., Application of a coupled Eulerian-Lagrangian method to simulate interactions between deformable composite structures and compressible multiphase flow. International journal for numerical methods in engineering, 80(12), pp. 1497-1519, 2009.

[26] Liu, Z., Young, Y.L., \& Motley, M.R., Transient response of partially-bonded sandwich plates subject to underwater explosions. Shock and Vibration ,17(3), pp. 233250, 2010.

[27] Son, G. \& Hur, N., A coupled level set and volume-of-fluid method for the buoyancydriven motion of fluid particles. Numerical Heat Transfer: Part B: Fundamentals, 42(6), pp. 523-542, 2002.

[28] Bergant, A., Simpson, A.R. \& Tijsseling, A.S., Water hammer with column separation: A historical review. Journal of fluids and structures, 22(2), pp. 135-171, 2006.

[29] Motley, M.R. \& Young, Y.L., Performance-based design and analysis of flexible composite propulsors. Journal of Fluids and Structures, 27(8), pp. 1310-1325, 2011. 